\title{
A Modified Dual-Wavelength Technique for Ku- and Ka-Band Radar Rain Retrieval
}

\author{
LIANG LIAO \\ Goddard Earth Science Technology and Research, Morgan State University, Columbia, Maryland \\ ROBERT MENEGHINI \\ NASA Goddard Space Flight Center, Greenbelt, Maryland
}

(Manuscript received 6 February 2018, in final form 5 November 2018)

\begin{abstract}
To overcome a deficiency in the standard $\mathrm{Ku}$ - and Ka-band dual-wavelength radar technique, a modified version of the method is introduced. The deficiency arises from ambiguities in the estimate of the massweighted diameter $D_{m}$ of the raindrop size distribution (DSD) derived from the differential frequency ratio (DFR), defined as the difference between the radar reflectivity factors (dB) at $\mathrm{Ku}$ and $\mathrm{Ka}$ band $Z_{\mathrm{Ku}}-Z_{\mathrm{Ka}}$. In particular, for DFR values less than zero, there are two possible solutions of $D_{m}$, leading to ambiguities in the retrieved DSD parameters. It is shown that the double solutions to $D_{m}$ are effectively eliminated if the DFR is modified from $Z_{\mathrm{Ku}}-Z_{\mathrm{Ka}}$ to $Z_{\mathrm{Ku}}-\gamma Z_{\mathrm{Ka}}(\mathrm{dB})$, where $\gamma$ is a constant with a value less than 0.8 . An optimal radar algorithm that uses the modified DFR for the retrieval of rain and $D_{m}$ profiles is described. The validity and accuracy of the algorithm are tested by applying it to radar profiles that are generated from measured DSD data. Comparisons of the rain rates and $D_{m}$ estimated from the modified DFR algorithm to the same hydrometeor quantities computed directly from the DSD spectra (or the truth) indicate that the modified DFR-based profiling retrievals perform fairly well and are superior in accuracy and robustness to retrievals using the standard DFR.
\end{abstract}

\section{Introduction}

There is an increasing trend of using multiple-wavelength radars in an effort to accurately detect and retrieve cloud and precipitation microphysical properties. The dualwavelength radar is, however, the most popular among these applications. It makes use of two wavelengths, one of which is such that Rayleigh scattering dominates for most precipitation-sized particles and the other short enough so that non-Rayleigh (or Mie) scattering occurs in the presence of large hydrometeors. For precipitation measurements, the combination of $\mathrm{Ku}$ (or $\mathrm{X}$ ) and $\mathrm{Ka}$ band is found in many air/spaceborne radar applications. This choice is dictated not only by the desire for microphysical information but by practical considerations such as frequency allocation (for space-based radars), the advantages of matched radar beams, and the desire for good spatial resolution within the constraints of antenna size. Attenuation caused by rain and mixed-phase

\footnotetext{
Corresponding author: Dr. Liang Liao, liang.liao-1@nasa.gov
}

hydrometeors, among other factors, is also important in choosing frequencies not only because it adds difficulty in estimating precipitation but because it often prevents the radar signal from penetrating the entire storm.

The dual-wavelength precipitation radar (DPR), operating at $\mathrm{Ku}$ and $\mathrm{Ka}$ bands aboard the Global Precipitation Measurement (GPM) core satellite, is the first dualwavelength radar in space (Hou et al. 2014). An important goal of the DPR is to derive rain rate and raindrop size distribution (DSD), the latter of which is often modeled by an analytical function (Ulbrich 1983; Gorgucci et al. 2000, 2002; Bringi et al. 2002; Feingold and Levin 1986). Several dual-wavelength radar retrieval techniques have been developed that are based on the standard dualwavelength technique that uses the differential frequency ratio (DFR; i.e., the difference of radar reflectivities between two wavelengths) to first infer the DSD parameters, then correct attenuation, and then derive the rain-rate profile gate by gate either stepping forward or backward along the radar beam (Meneghini et al. 1992, 1997; Mardiana et al. 2004; Liao and Meneghini 2005; 
Rose and Chandrasekar 2005; Seto et al. 2013; Seto and Iguchi 2015; Liao et al. 2016). To improve the robustness of the dual-wavelength retrieval, several optimal estimation methods have been recently proposed, including the GPM DPR (version 5) algorithm in which one or more adjustment factors are used to modify nominal relationships between the parameters of the radar and the precipitation (such as $k-Z$ and $R-D_{m}$ relations, where $k, Z, R$, and $D_{m}$ are specific attenuation, radar reflectivity, rain rate, and mass-weighted diameter) to account for variations in the DSD in space and time (Grecu et al. 2011; Seto and Iguchi 2015). Adjustment factors at each gate/profile are determined by optimizing predefined cost functions that constrain the values of the reflectivities and path integral attenuations (PIA).

In the standard DFR-based dual-wavelength technique, the two unknown DSD parameters, related to the characteristic size and number density of raindrops, are estimated at each range gate. However, this is successful only if there exist unique solutions of the DSD parameters to the dual-wavelength radar equations. For the gamma DSD model, ambiguities in the estimation of $D_{m}$ often occur, particularly for light-to-moderate rain rates. As there is no additional information aiding in selection of the solution, the standard dual-wavelength technique becomes problematic for rain retrievals. Retrieval errors caused by improper selection of the solution worsen as retrieval proceeds deep into the storm as a result of error propagation. It has been revealed in our study from a large collection of the measured DSD data from various NASA-sponsored field campaigns that negative DFR values occur frequently. Since these negative DFR values lead to ambiguities in the estimate of $D_{m}$ and therefore $R$, and further that these errors are propagated in range because of attenuation correction, the overall performance of the dual-frequency retrieval will be adversely affected.

To circumvent the double-value problem of $D_{m}$, a modified DFR, denoted by DFR*, is introduced in this study. Instead of weighting equally the $\mathrm{Ku}$ - and $\mathrm{Ka}$-band reflectivities, the DFR* weights the Ka-band reflectivity less than the Ku-band reflectivity so that the possibility of double solutions diminishes. Removal of the double values of $D_{m}$ with use of DFR* aids in finding $D_{m}$ unambiguously from the $D_{m}-\mathrm{DFR} *$ relations; the tradeoff, however, is that the $\mathrm{DFR}^{*}$ is no longer exclusively a function of $D_{m}$ but a function of the number concentration parameter as well.

In this study, our focus is on examining the potential of DFR* for the retrieval of the vertical rain and DSD profiles by employing measured DSD data. For understanding the uncertainties in rain-rate estimation and also in gaining insight into ways to improve the algorithms, a physical evaluation of the standard (DFR based) and modified (DFR* based) dual-wavelength techniques is conducted by applying them to simulated vertical rain profiles. The hydrometeor profiles for use in our evaluation are constructed by using the measured DSD acquired from various storm systems.

In this paper, we begin with briefly describing the principle and issues associated with the standard dualwavelength technique in section 2 , followed by the discussion of the modified dual-wavelength technique in section 3. Described in section 4 are the methodology related to the radar profiling algorithms and their procedures for the retrieval of rain and DSD profiles using DFR and DFR* while evaluation of the performance of the profiling algorithms is made in section 5. Summary and remarks are given in section 6 .

\section{Standard dual-wavelength radar technique}

The measured radar reflectivity factor $\left(z_{m} ; \mathrm{mm}^{6} \mathrm{~m}^{-3}\right)$ of the hydrometeors at range $r$ and at wavelength $\lambda$ can be expressed as

$$
z_{m}=z_{e} \exp \left[-0.2 \ln (10) \int_{0}^{r} k d r\right],
$$

where $z_{e}$ is the effective radar reflectivity factor $\left(\mathrm{mm}^{6} \mathrm{~m}^{-3}\right)$ and $k$ is specific attenuation $\left(\mathrm{dB} \mathrm{km}^{-1}\right)$, expressed as

$$
\begin{gathered}
z_{e}=\frac{\lambda^{4}}{\pi^{5}\left|K_{w}\right|^{2}} \int_{0}^{\infty} N(D) \sigma_{b}(D, \lambda) d D, \text { and } \\
k=4.343 \times 10^{-3} \int_{0}^{\infty} N(D) \sigma_{e}(D, \lambda) d D,
\end{gathered}
$$

where $\sigma_{b}(D, \lambda)$ and $\sigma_{e}(D, \lambda)$ are radar backscattering and extinction cross sections $\left(\mathrm{mm}^{2}\right)$ of a raindrop with volume equivalent diameter $D(\mathrm{~mm})$ at a wavelength of $\lambda(\mathrm{mm})$, respectively. $N(D)\left(\mathrm{mm}^{-1} \mathrm{~m}^{-3}\right)$ is the gamma DSD and expressed as (Ulbrich 1983; Bringi and Chandrasekar 2001)

$$
N(D)=N_{w} f(\mu)\left(\frac{D}{D_{m}}\right)^{\mu} \exp (-\Lambda D),
$$

where $N_{w}$ is a scale factor, and

$$
\begin{aligned}
f(\mu) & =\frac{6(4+\mu)^{\mu+1}}{4^{4} \Gamma(\mu+4)}, \quad \text { and } \\
\Lambda & =(4+\mu) / D_{m},
\end{aligned}
$$

where $\Gamma$ is the Gamma function, and $\mu$ is the shape factor of the gamma distribution. The term $D_{m}$ is the 

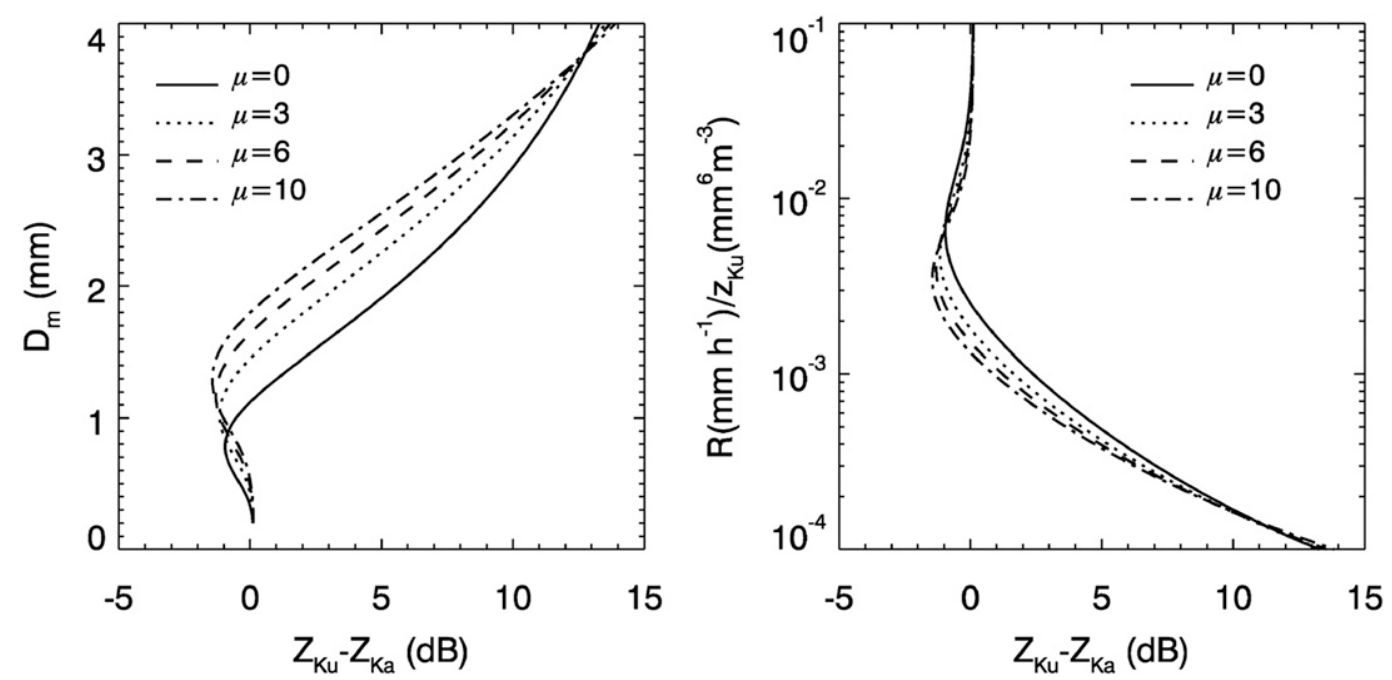

FIG. 1. Computational results of (left) $D_{m}$ vs DFR and (right) $z_{\mathrm{Ku}}$-normalized rain rate vs DFR as the gamma DSD model is assumed with $\mu$ values of $0,3,6$, and 10 .

mass-weighted diameter of particle spectra, defined as follows:

$$
D_{m}=\frac{\int_{D_{\min }}^{D_{\max }} D^{4} N(D) d D}{\int_{D_{\min }}^{D_{\max }} D^{3} N(D) d D},
$$

where $D_{\min }$ and $D_{\max }$ are the minimum and maximum diameters of raindrops, respectively. The rain rate is computed by

$$
R=6 \pi \times 10^{-4} \int_{D_{\min }}^{D_{\max }} N(D) D^{3} V(D) d D,
$$

where $V(D)$ is raindrop fall velocity and expressed as a function of $D$.

Because the radar reflectivity factor usually spans a large range of magnitudes, it is conveniently expressed in decibels of reflectivity $(\mathrm{dB} Z)$ as follows:

$$
Z=10 \log _{10} z
$$

Note that lowercase $z$ and uppercase $Z$ denote linear and logarithmic reflectivities, respectively, throughout this study. The DFR (dB) is defined as

$$
\mathrm{DFR}=Z_{\mathrm{Ku}}-Z_{\mathrm{Ka}}
$$

where $Z_{\mathrm{Ku}}(\mathrm{dB} Z)$ and $Z_{\mathrm{Ka}}(\mathrm{dB} Z)$ are the radar reflectivity factors at wavelengths of the $\mathrm{Ku}$ and $\mathrm{Ka}$ bands. From (2), (4), and (10) it is not difficult to show that DFR is independent of $N_{w}$. Using (4), Fig. 1 shows $D_{m}$ (left) and the $z_{\mathrm{Ku}}$-normalized rain rate (right) as a function of DFR for several $\mu$ values. Note that the oneto-one relationship between $D_{m}$ and DFR when DFR is greater than zero, implying that, if $\mu$ is known and the DFR is positive, $D_{m}$ can be determined uniquely. However, double solutions of $D_{m}$ occur as the DFR becomes negative. This has also been found by many earlier studies (Meneghini et al. 1992, 1997; Mardiana et al. 2004; Liao and Meneghini 2005; Rose and Chandrasekar 2005; Seto et al. 2013; Seto and Iguchi 2015). Similar features are found in the normalized rain-rate plots shown in the right panel of Fig. 1, where a unique solution to $R / z_{\mathrm{Ku}}$ is available only when the DFR is positive.

The axes of the plots in Fig. 2 are the same as in Fig. 1 but in this case, the results, displayed as $2 \mathrm{D}$ probability density functions (PDF), are derived from measured DSDs acquired during the Iowa Flood Studies (IFloodS), the Integrated Precipitation and Hydrology Experiment (IPHEx), and data from the NASA Wallops Flight Facility in Wallops Island, Virginia. For the sake of comparisons with model results, theoretical computations from the gamma DSD model with a fixed $\mu$ of 3 are given by the white curves. It is worth mentioning that use of the $z_{\mathrm{Ku}}$-normalized rain rate, as in Figs. 1 and 2, is a convenient and effective way to directly infer rain rate from radar parameters. In particular, given a (positive) DFR and an assumed $\mu$, the $R / z_{\mathrm{Ku}}$ is obtained from the curves in the figure; multiplication of the result by $z_{\mathrm{Ku}}$ then provides the rain-rate estimate. The term $N_{w}$ is obtained from either $\mathrm{Ku}$ - or Ka-band reflectivities along with the derived $D_{m}$. For the scattering computations, the raindrops are prescribed as horizontally oriented oblate spheroids with axis ratios that follow the shape-size relations reported 

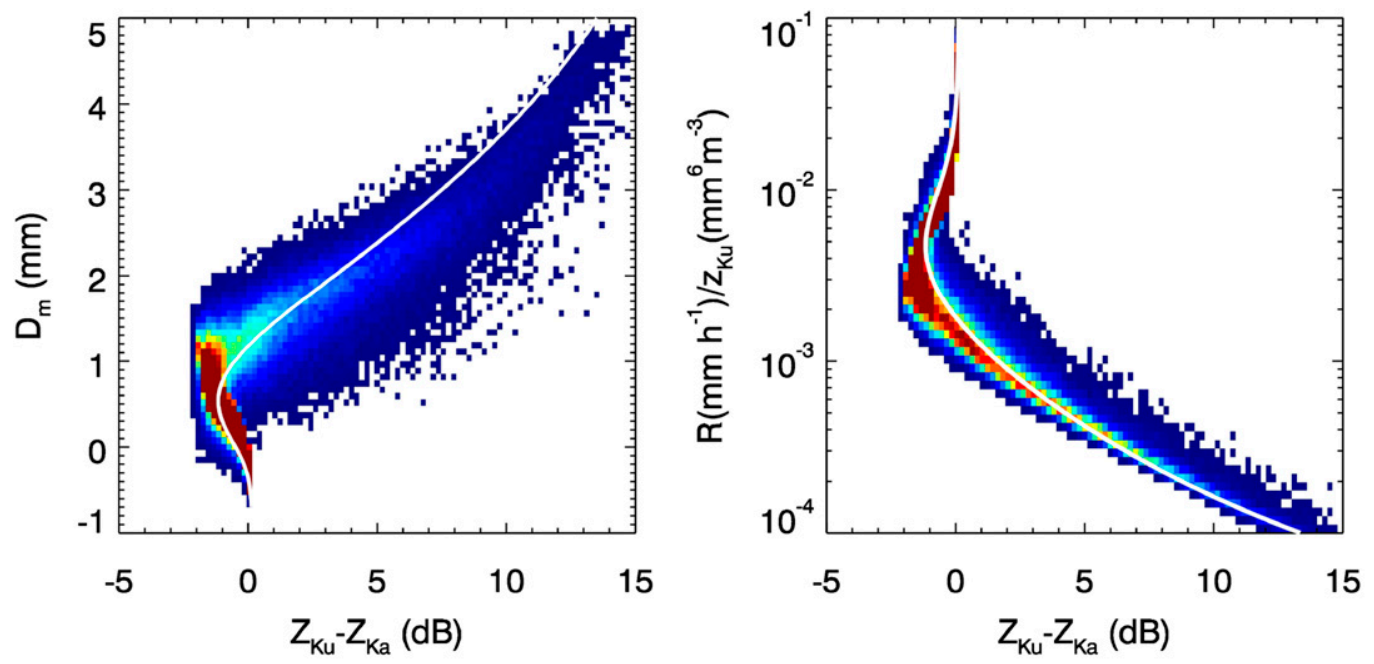

\subsection{0}

0.04

0.08

0.12

0.16

0.20

FIG. 2. The 2D probability density functions, as derived from the DSD measurements taken during several NASA field campaigns, between (left) $D_{m}$ and DFR and (right) $z_{\mathrm{Ku}}$-normalized rain rate vs DFR. Theoretical computations from the gamma DSD model of $\mu=3$ are also plotted as references.

by Thurai et al. (2007). Symmetrical axes of the raindrops are aligned in the vertical direction. The T-matrix method is used to compute the scattering properties of single particles (Mishchenko and Travis 1998). It is important to point out that vast majority of the DSD measurements (nearly $70 \%$ ), as found in Fig. 2, fall into the range where the DFR is negative, which suggests a serious problem for operational retrieval algorithms if the DFR $-D_{m}$ relations are used. For the GPM DPR whose minimum detectable reflectivities are approximately 12 and $17 \mathrm{~dB}$ at $\mathrm{Ku}-$ and Ka-wavelengths, respectively, the percentage of the DSD data with the DFR less than 0 is around $37 \%$.

\section{Modified dual-wavelength radar technique}

As discussed above, the standard dual-wavelength algorithm is capable of retrieving two parameters of the DSD and rain rate at each range gate if the fixed$\mu$ gamma DSD model is assumed and if the DFR is positive along the path. Apart from errors in the DSD model assumed, the most serious problem arises from ambiguities in the DFR $-D_{m}$ and DFR $-R / z_{\mathrm{Ku}}$ relationships when the DFR is negative. To lessen the impact of these ambiguities, a modified DFR, denoted by DFR*, is introduced and expressed as

$$
\mathrm{DFR}^{*}=Z_{\mathrm{Ku}}-\gamma Z_{\mathrm{Ka}}
$$

where $\gamma$ is a scale factor with a value ranging from 0 to 1 . The definition of DFR* in (11) can be viewed as a generalized form of the dual-wavelength ratio that reverts to the single-wavelength $Z_{\mathrm{Ku}}$ when $\gamma=0$ and to the standard dual-wavelength method when $\gamma=1$. For $\gamma$ greater than 0 but less than 1, the Ka-band radar reflectivity is used but reduced from that in the DFR relation. Unlike the DFR, the DFR* is dependent on $N_{w}$.

Figure 3 illustrates the $\mathrm{DFR}^{*}-D_{m}$ relation, derived from the DSD data, as $\gamma$ takes on different values. The thick black solid lines are the means of the data taken at each point along the horizontal axis. Theoretical computations (blue curves) derived from the gamma DSD model with a fixed $\mu$ of 3 are plotted for $N_{w}$ varying from 10 to $10^{5} \mathrm{~mm}^{-1} \mathrm{~m}^{-3}$. For reference, the contours of $Z_{\mathrm{Ku}}(\mathrm{dBZ}$; red curves) are also given in the plots. Shown in the topleft panel is the case for the single $\mathrm{Ku}$ band (i.e., $\gamma=0$ ), while the results from the standard dual-wavelength technique $(\gamma=1)$ are shown in the bottom-right panel. The cases where $\gamma$ is equal to 0.5 and 0.7 are displayed in the top-right and bottom-left panels, respectively. Several interesting features are revealed in Fig. 3; in particular, $D_{m}$ has a one-to-one relation with $\mathrm{DFR}^{*}$ for all the cases shown except for the case where $\gamma=1$. As expected, the $\mathrm{DFR}^{*}-D_{m}$ relations depend on $N_{w}$ if $\gamma$ is not 1 , and the degree of the dependence depends on $\gamma$. The strongest dependence of $N_{w}$ on these relations occurs at $\gamma=0$ (Ku band only), as shown from both the DSD-derived 

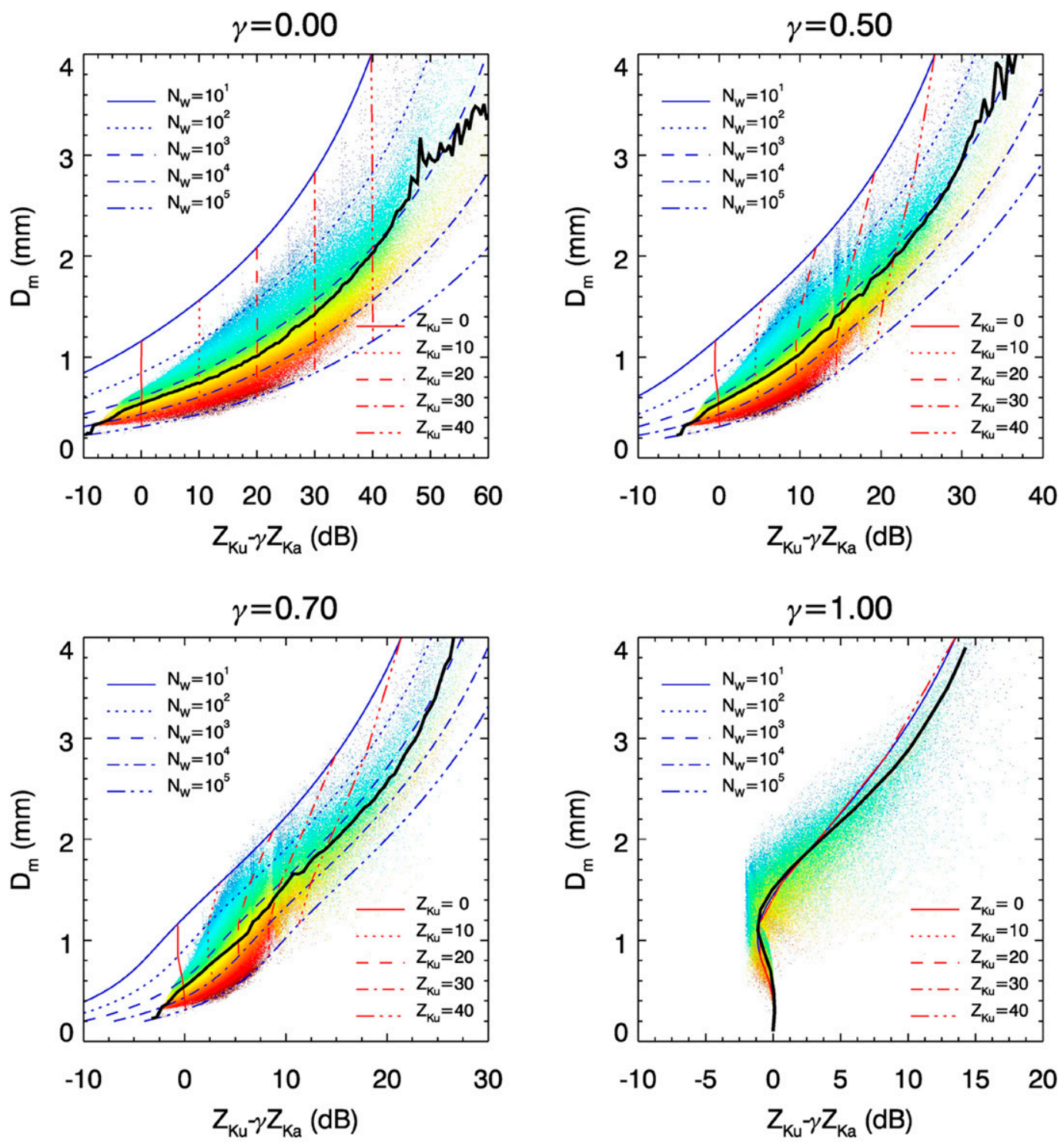

$\log _{10} \mathrm{~N}_{\mathrm{w}}$

1

2

3

4

5

FIG. 3. Scatterplots of $D_{m}$ vs modified DFR as derived from the DSD data for the cases of the weighting factor $\gamma$ of the modified DFR equal to $0,0.5,0.7$, and 1 . Colors of the data points depict $N_{w}$ values that are derived from the same DSD data. The mean values of the data are given by the thick dark curves. Theoretical model computations, represented by blue and red curves, are provided as the DSD is modeled as gamma distributions with a fixed $\mu$ of 3 and $N_{w}$ (blue curves) ranging from 10 to $10^{5} \mathrm{~mm}^{-1} \mathrm{~m}^{-3}$. Contours of $Z_{\mathrm{Ku}}$ at the values from 0 to $40 \mathrm{~dB}$ (red curves) are plotted as well for reference.

data points and the model computations. Variability in the DFR $*-D_{m}$ with respect to $N_{w}$ tends to decrease for $D_{m}$ greater than $1 \mathrm{~mm}$ as $\gamma$ increases. The DFR* at $\gamma=1$, or the standard DFR, is independent of $N_{w}$. It is worth reiterating that one of the crucial differences between the $\mathrm{DFR}^{*}(\gamma \neq 1)$ and the standard DFR is that the former gives a unique solution to $D_{m}$ for given $N_{w}$ and $\mu$, while the latter results in double values of $D_{m}$ for large amount of the radar measurements. Similar behavior is also found in the rain-rate estimates, as depicted in Fig. 4. Unique solutions to $D_{m}$ and rain rate from the DFR* are perhaps the most desirable features from the perspective of radar 

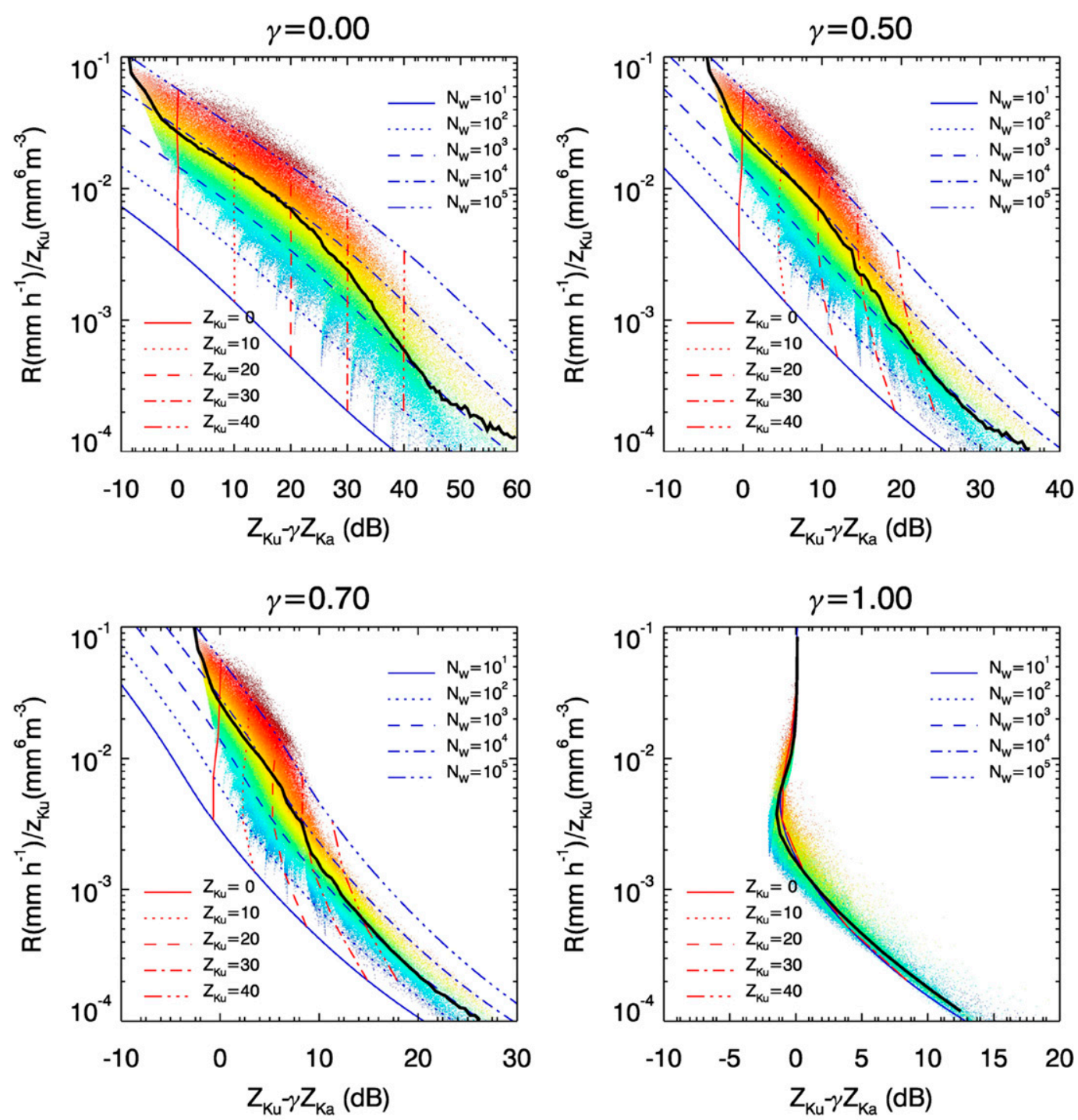

$\log _{10} N_{w}$

1

2

3

4

FIG. 4. Scatterplots of $Z_{\mathrm{Ku}}$-normalized rain rate vs modified DFR as derived from the DSD data for the cases of the weighting factor $\gamma$ of the modified DFR equal to $0,0.5,0.7$, and 1 . Colors of the data points depict $N_{w}$ values derived from the same DSD data. The mean values of the data are given by the thick dark curves. Theoretical model computations, represented by blue and red curves, are provided as the DSD is modeled as gamma distributions with a fixed $\mu$ of 3 and $N_{w}$ ranging from 10 to $10^{5} \mathrm{~mm}^{-1} \mathrm{~m}^{-3}$. Contours of $Z_{\mathrm{Ku}}$ at the values from 0 to $40 \mathrm{~dB}$ (red curves) are plotted as well for reference.

retrievals, but it comes with the cost of adding the variable $N_{w}$ to the equations and leading to an underconstrained retrieval. As one of the goals of the spaceborne radar is to obtain hydrometeor profiles along the radar beam, the rest of the paper is focused on how the modified dualwavelength technique described above is implemented in the profiling algorithms and how its performance compares with algorithms that rely on the standard dualwavelength technique.

\section{Hydrometeor profile retrieval}

Although the modified dual-wavelength method or DFR* shows potential to improve accuracy of radar 
estimates over the standard DFR in terms of the uncertainties associated with double solutions, a physical evaluation of the hydrometeor profiling retrieval is required not only for understanding the uncertainties but also in gaining insight into ways to improve the retrievals. To achieve this, the measured DSD data acquired from a variety of storm systems during the NASA field campaigns are used to construct realistic hydrometeor profiles. Using measured DSD spectra, both radar and hydrometeor's parameters, which include true and measured radar reflectivities, attenuation, rain, and DSD characteristic parameters $\left(D_{m}\right.$ and $\left.N_{w}\right)$, can be computed. These DSD-calculated parameters then serve as truth. An evaluation of algorithm performances is actually conducted by comparing the radar estimates with the truth. Before showing the results on the DFR* profiling algorithm performance, we will first describe the radar and hydrometeor profiles simulated by using measured DSD and then detail the procedures of the DFR* profile algorithms.

\section{a. DSD profiles}

For simplicity but without losing generality, two types of vertical DSD profiles are created: One type is the vertically uniform DSD profile where a single DSD spectrum is used for entire column, and the second type is the nonuniform profile in which the DSD changes gate by gate along the column. The DSD data used in this study are primarily from measurements made by Particle Size Velocity (PARSIVEL) disdrometers during the IFloodS field experiment from 1 May to 15 June 2013, IPHEx from 1 May to 15 June 2014, and the observations at the NASA Wallops Flight Facility in Wallops Island, Virginia, from 2013 to 2014. The PARSIVEL disdrometers are used to measure rain DSD and fall velocities of particle sizes from 0.3 to $20 \mathrm{~mm}$.

Shown in Fig. 5 is an example of the simulated Kuband radar reflectivities (top panels) and rain rates (bottom panels) from a segment of 200 DSD vertically uniform (left column) and nonuniform (right column) profiles. The range resolution is $0.125 \mathrm{~km}$, which is comparable to the case for the GPM DPR, with the rain height set at $5 \mathrm{~km}$. To construct realistic nonuniform hydrometeor profiles, the time series DSD data are converted to vertical profiles by assigning 1-minintegration-time DSD data to the gates from the top to the surface consecutively. The mean values of the correlations of rain, $D_{m}$, and $\log _{10} N_{w}$ over all simulated nonuniform profiles between the range gates considered to the surface gate are plotted in Fig. 6 for the cases in which rain rate is less than $5 \mathrm{~mm} \mathrm{~h}^{-1}$ (left), greater than $5 \mathrm{~mm} \mathrm{~h}^{-1}$ (center), and greater than zero (right), which approximately represent the stratiform, convective, and stratiform-convective combined rain systems. These correlations are found to be comparable to the results reported by Adirosi et al. (2016) based on a combined study of Micro Rain Radar (MRR) and DSD measurements.

\section{b. Modified DFR profiling algorithms}

As shown in Fig. 3, $D_{m}$ can be obtained from DFR* if $N_{w}$ is known. Likewise, rain rate can be derived if DFR* is used along with $Z_{\mathrm{Ku}}$, as shown in Fig. 4. These procedures for the retrieval of $D_{m}$ and rain rate constitute the basis for the DFR*-based profiling algorithms. On the other hand, the Ku- and Ka-band radar signals undergo losses caused by attenuation of the signals out to the gate at which the radar measurements are made. Accurate attenuation correction is therefore important for the algorithm's overall performance. For downlooking air/spaceborne radar, forward and backward recursive procedures are often used for attenuation correction. In the forward approach, the retrieval starts from storm top and then proceeds into storm gate by gate until reaching the surface or final clutter-free gate. Conversely, the backward approach begins at the surface with a known PIA that is usually estimated by the surface reference technique (SRT) or other independent measurements (such as radiometers), with the correction moving upward until reaching the storm top. Benefits and demerits of the forward and backward approaches depend on the types of error sources involved in the algorithms. Nevertheless, it is clear that uncertainties associated with PIA only affect the performance of the backward recursive method, while errors associated with attenuation corrections prior to rain, such as attenuation resulting from cloud water and the melting region, are directly related to the performance of the forward recursive method.

To retrieve rain and DSD profiles using DFR*, one simple way is to assign the same $N_{w}$ to each range gate of one vertical profile as an initialization process. With a known $N_{w}, D_{m}$ can be uniquely derived from the DFR* if a fixed $\mu$ is assumed; rain rate and attenuation coefficients can then be derived by using DFR* along with $Z_{\mathrm{Ku}}$. Attenuation corrections to the measured radar reflectivity profiles are carried out consecutively gateby-gate along the radar beam either in the forward or backward direction. Since the derived hydrometeor profiles depend on the value of $N_{w}$ assumed, finding the proper $N_{w}$ that leads to accurate DSD and rain profiles is a key to the retrieval procedure.

In the search for $N_{w}$, an optimization method is employed to minimize the discrepancies between the simulated and measured radar parameters. Following Seto et al. (2016), $N_{w}$ is selected to maximize the product of the following three probability functions (or constraints) 

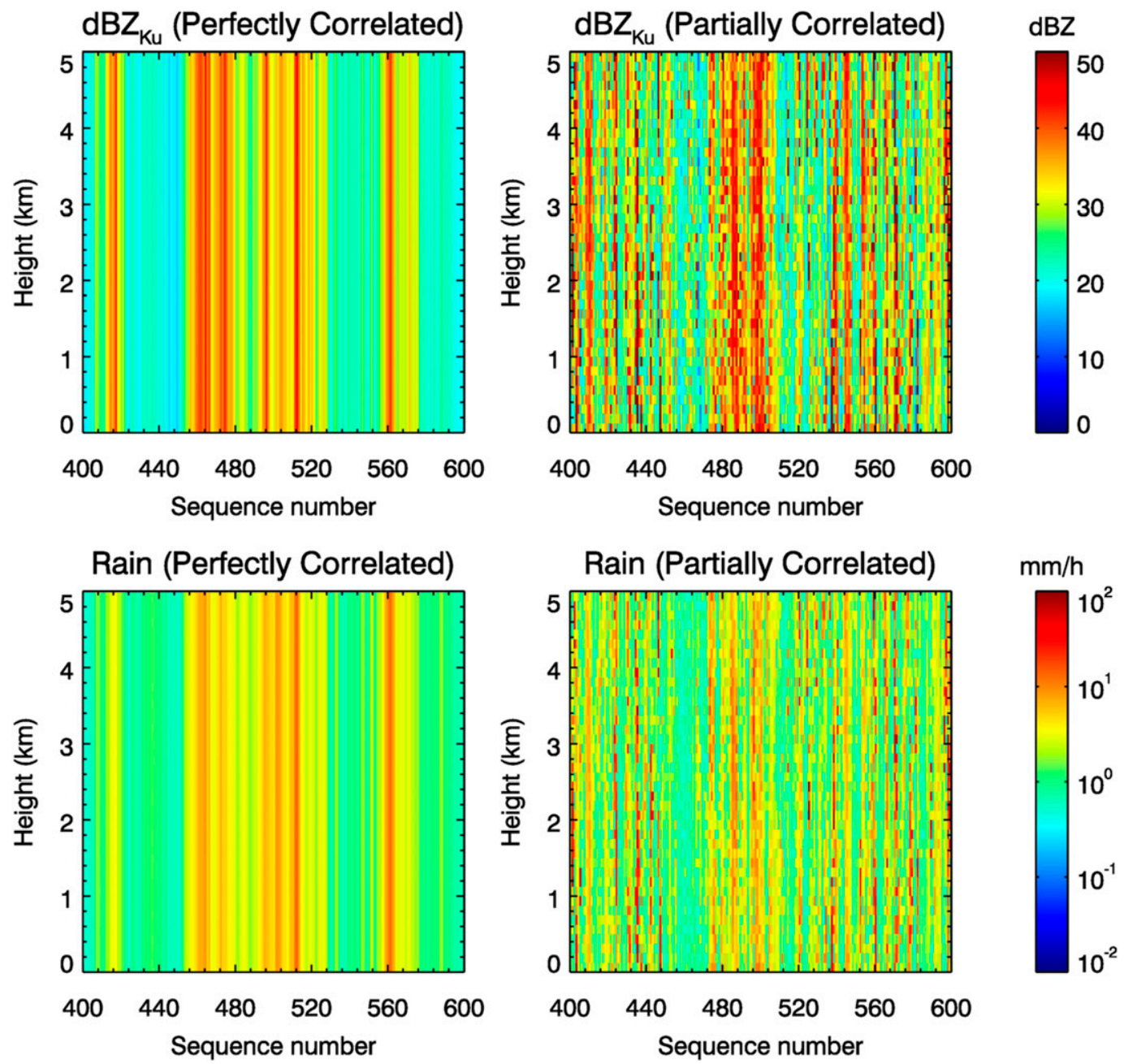

FIG. 5. (top) Ku-band radar reflectivities and (bottom) rain rate simulated from a segment of the DSD profiles generated from measured DSD data. (left) Homogeneously vertical DSD profiles; (right) nonuniform DSD profiles.

from the computations conducted by using a series of initial values of $N_{w}$, denoted by $\tilde{N}_{w, k}$, where $k=1,2, \ldots$, $K$ (where $K$ is the total number of the initial tests of $N_{w}$ ),

$p_{1}\left(N_{w}\right) p_{2}\left(N_{w}\right) p_{3}\left(N_{w}\right)=\max \left[p_{1}\left(\tilde{N}_{w, k}\right) p_{2}\left(\tilde{N}_{w, k}\right) p_{3}\left(\tilde{N}_{w, k}\right)\right]$,

where

$$
\begin{aligned}
& p_{1}\left(\tilde{N}_{w, k}\right)=\frac{1}{\sigma_{1} \sqrt{2 \pi}} \exp \left[-\frac{\left(\log _{10} \tilde{N}_{w, k}-3.45\right)^{2}}{2 \sigma_{1}^{2}}\right], \\
& p_{2}\left(\tilde{N}_{w, k}\right)=\frac{1}{\sqrt{2 \pi}} \exp \left\{-\frac{\left[\delta \mathrm{PIA}\left(\tilde{N}_{w, k}\right)-\delta \mathrm{PIA}_{\mathrm{SRT}}\right]^{2}}{2 \sigma_{2}^{2}}\right\},
\end{aligned}
$$

and
$p_{3}\left(\tilde{N}_{w, k}\right)=\prod_{n=1}^{N} \frac{1}{\sqrt{2 \pi}} \exp \left\{-\frac{\left[Z_{m, n, \text { est }}^{(\mathrm{Ka})}\left(\tilde{N}_{w, k}\right)-Z_{m, n, \mathrm{obs}}^{(\mathrm{Ka})}\right]^{2}}{2 N \sigma_{3}^{2}}\right\}$

where $N$ is the number of range gates along the column, and $\delta$ PIA is the differential PIA over the full column defined as difference of PIA between Ka and Ku band. $Z_{m, n, \text { est }}^{(\mathrm{Ka})}\left(\tilde{N}_{w, k}\right)$ and $Z_{m, n, \text { obs }}^{(\mathrm{Ka})}$ are respectively the simulated and measured Ka-band apparent radar reflectivities at the nth range gate. The function $p_{1}\left(\tilde{N}_{w, k}\right)$ describes the distribution of $\log _{10}\left(N_{w}\right)$ that has a mean of $3.45 \log _{10}\left(\mathrm{~mm}^{-1} \mathrm{~m}^{-3}\right)$ and a standard deviation $\sigma_{1}$, derived from the DSD data. The function $p_{2}\left(\tilde{N}_{w, k}\right)$ is associated with the difference in $\delta$ PIA between the DSD-estimated value and the SRT estimate while $p_{3}\left(\tilde{N}_{w, k}\right)$ accounts for discrepancies between the simulated and measured Ka-band reflectivities. 

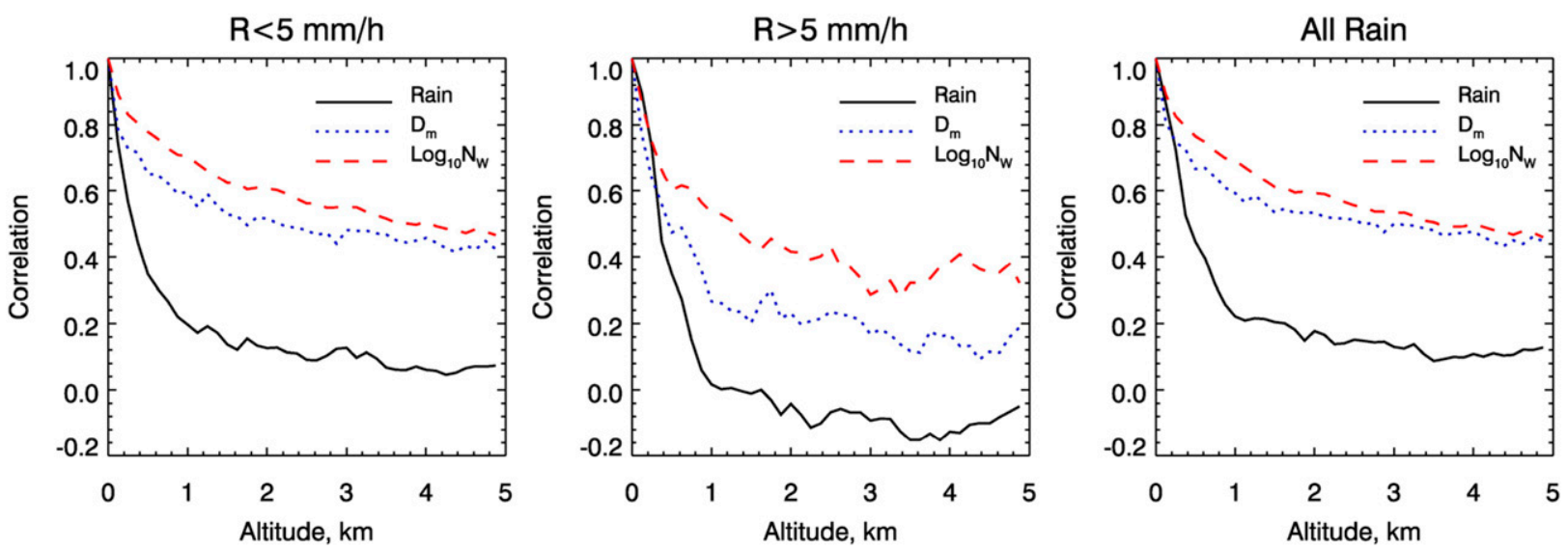

FIG. 6. Averaged spatial correlations of rain rate, $D_{m}$, and $\log _{10} N_{w}$ for the nonuniform DSD profiles between radar range heights and surface. Results from three rain ranges, i.e., $R<5, R \geq 5$, and $R>0 \mathrm{~mm} \mathrm{~h}^{-1}$ (all rain), are provided for approximately representing stratiform, convective, and their combined systems.

Note that $\left(\sigma_{2}\right)^{2}$ is the error variance associated with the SRT estimate of the differential path attenuation. The term $\sigma_{3}$ is a constant that determines weighting of measurements of the Ka-band reflectivities in the overall constraint. The smaller this value is, the more the Kaband measurements are weighted. It is worth mentioning that the reason that $\delta$ PIA is used rather than the PIA at either or both wavelengths is that the former usually has a smaller error variance than the latter if they are estimated by the SRT because of strong correlation of the normalized surface cross section $\left(\sigma^{0}\right)$ between $\mathrm{Ku}$ and $\mathrm{Ka}$ band (Meneghini et al. 2015). It is also worth noting that optimization is not required in the standard method since in this case, the DFR is used to estimate $D_{m}$ directly; $N_{w}$ is then found from $D_{m}$ and $Z_{\mathrm{Ku}}$ or $Z_{\mathrm{Ka}}$.

More detail on the optimization described above is given by the flowchart in Fig. 7, which shows the gateby-gate progression of the retrieval from the rain top to the surface (forward approach). The procedure starts with equally spaced values of $\log _{10}\left(\tilde{N}_{w, k}\right)(k=1,2, \ldots, K$, where $K=100$ ) in the range from 0 to 6 , from which the radar and hydrometeor profiles are estimated. The procedure is repeated $K$ times, and the final $N_{w}$ is chosen as the value that maximizes (12). Once $N_{w}$ is selected, profiles of $D_{m}$ and rain rate are found from lookup tables (LUT) that express $D_{m}$ as a function of $N_{w}$ and $\mathrm{DFR}^{*}(\gamma)$. Accumulated $\mathrm{Ku}-$ and Ka-band attenuations ( $A_{\mathrm{Ku}}$ and $\left.A_{\mathrm{Ka}} ; \mathrm{dB}\right)$ are computed from the specific attenuations $\left(k_{\mathrm{Ku}}\right.$ and $\left.k_{\mathrm{Ka}} ; \mathrm{dB} \mathrm{km}^{-1}\right)$ of previous gates. The backward approach is done in the same fashion. Unlike the forward approach where the retrieval starts at the rain top where the attenuation is assumed to be negligible, the backward approach begins at the gate near surface where the SRT-derived PIAs are used to estimate the attenuation-corrected reflectivities from the measured radar reflectivities. The backward retrievals are therefore dependent on the accuracy of the PIA estimate.

\section{Evaluation of DFR* algorithms}

Assessments of the modified DFR performance in estimates of rain and $D_{m}$ are conducted by comparing the estimated results with the true values that are obtained from direct computations from the simulated DSD profiles. Figures 8 and 9 depict, in terms of 2D probability density functions, the comparisons of $D_{m}$ and rain rates derived from the modified DFR with $\gamma=0$ and 0.7 (left and center columns) and the standard DFR (right columns) to the corresponding true values of $D_{m}$ and $R$. The forward recursive approach, as described in Fig. 7, is applied to the nonuniform DSD profiles that are prescribed earlier for these retrievals. To track the statistical errors along the profiles, the comparisons are made at the rain top and the surface that correspond to the beginning and end of the profiles. An unbiased $\delta$ PIA model with the standard deviation of $0.8 \mathrm{~dB}$, which is similar to what has been found from the GPM DPR measurements over ocean, is used to characterize the PIA errors. The $\sigma_{1}, \sigma_{2}$, and $\sigma_{3}$ in (13)-(15) are $3.45 \log _{10}\left(\mathrm{~mm}^{-1} \mathrm{~m}^{-3}\right), 1.6 \mathrm{~dB}(2$ times the standard deviation of $\delta$ PIA) and $2 \mathrm{~dB} Z$, respectively. The choice of these values seems to lead to reasonable and robust estimates after extensive tests. For the standard DFR method, when negative DFR occurs, the larger of the two $D_{m}$ solutions is chosen. To be consistent with the GPM DPR, the DSD data are used only if the DSD-computed Ku-band reflectivities are greater than $12 \mathrm{dBZ}$ and the Ka-band reflectivities are greater than $17 \mathrm{dBZ}$ (DPR sensitivity thresholds). Comparisons of the results derived from the modified and standard DFR methods clearly indicate that 


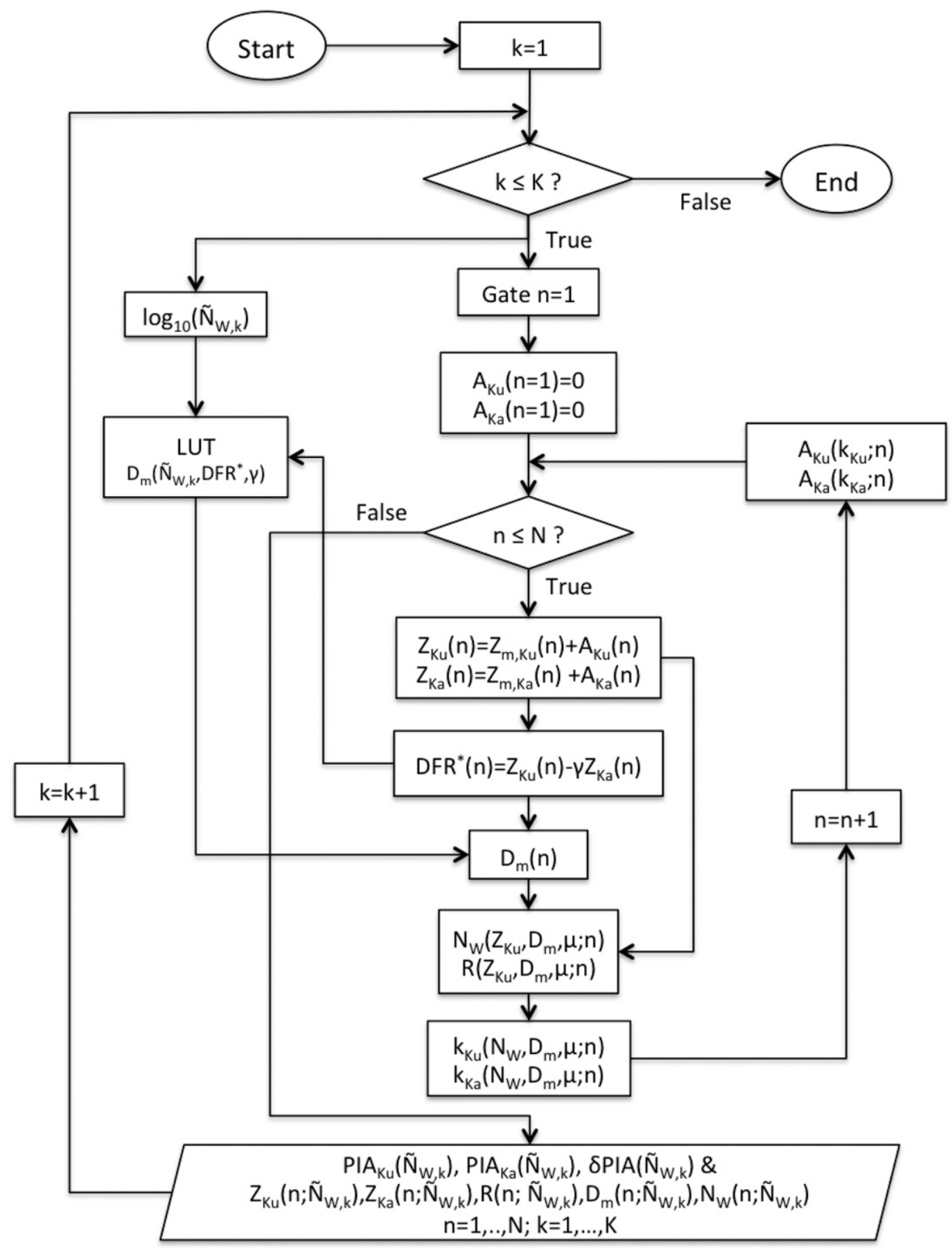

FIG. 7. Flowchart of DFR* for retrieval of DSD parameters and rain rate. Outer-loop variable $k$ goes from 1 to $K$ to provide equal-spaced values of $\log _{10}\left(\tilde{N}_{w, k}\right)$ at each step, while inner-loop variable $n$, running from 1 to $N$, represents radar range gates at which the retrievals are carried out.

the modified DFR delivers far more accurate estimates than does the standard DFR for both rain rate and $D_{m}$. The results show generally good correlations between the modified-DFR estimates and the true values despite a slight degradation in accuracy as the retrieval progresses down to the surface. This degradation is the result of accumulating errors in the attenuation correction for ranges deeper into the storm. The rain estimates are more accurate than the $D_{m}$ estimates because retrieval of $D_{m}$ is more sensitive to the DSD model assumed, such as selection 

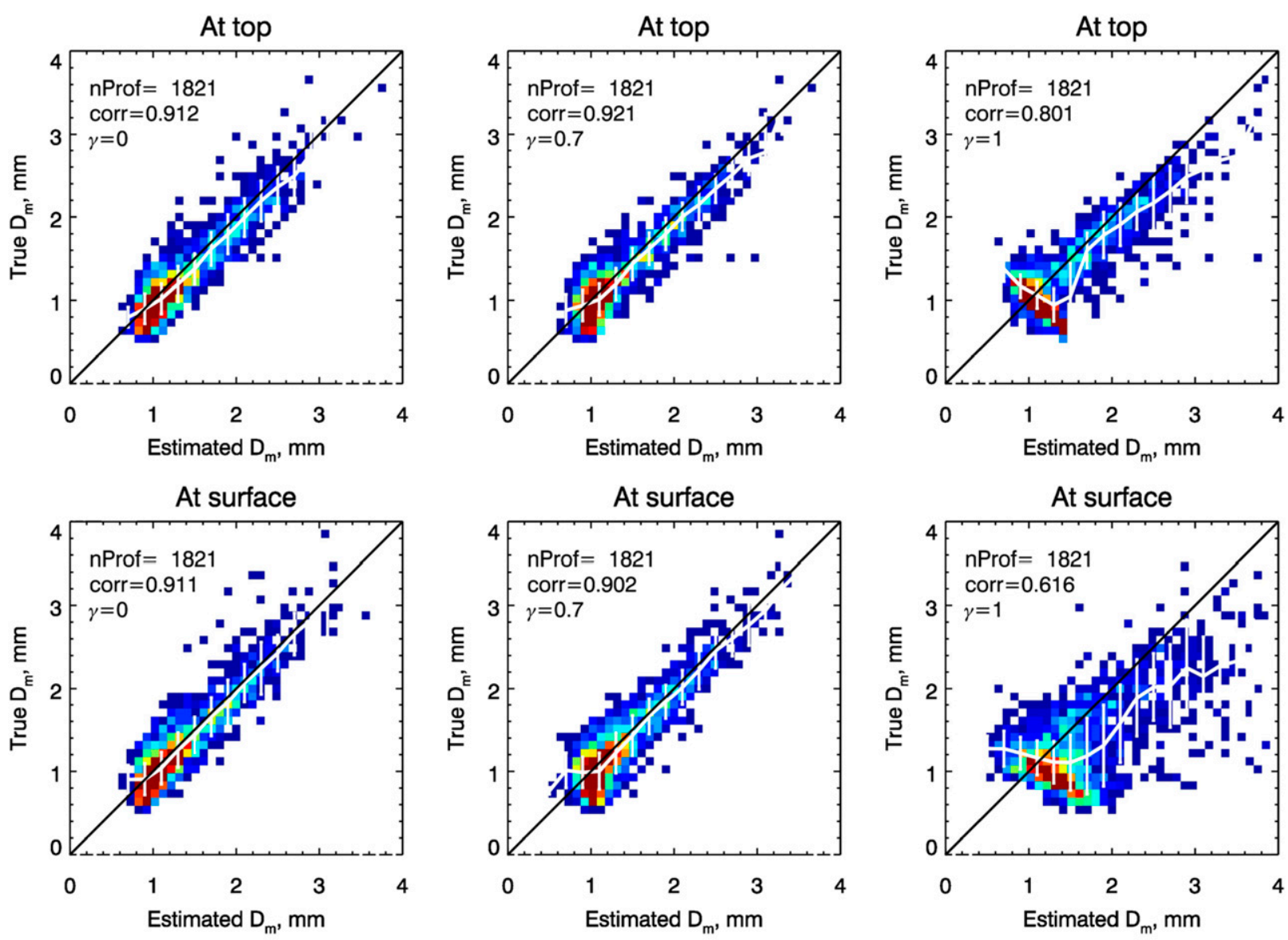

PDF

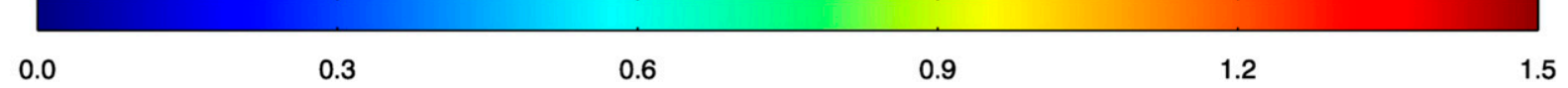

FIG. 8. The 2D probability density functions of $D_{m}$ estimated by the modified DFR with $\gamma$ of (left) 0 and (center) 0.7 as well as (right) the standard DFR ( $\gamma=1$ ) with the true $D_{m}$ for the range gate at (top) the rain top and (bottom) the surface. The one-to-one lines are given by the black solid curves, while the means and 2 times the standard deviations of the data are given by the white solid curves and vertical bars, respectively. For both the modified and standard DFR methods, the forward recursive approach is used and applied to the nonuniform DSD profiles. An unbiased statistical $\delta$ PIA model with the standard deviation of $0.8 \mathrm{~dB}$ is assumed.

of $\mu$ for the gamma distribution, than is the rain retrieval. It is noticed that there are only slight differences of $R$ and $D_{m}$ estimates between the $\gamma$ values of 0 and 0.7 . This is because the nonuniform DSD profiles presented in this study do not actually result in significantly large variations of $N_{w}$ in the profiles. For the case of relatively highly uncorrelated DSD profile, in which the differences in the estimates between $\gamma=0$ and $\gamma=0.7$ are clearly noticeable; that is, the estimates at $\gamma=0.7$ appear less variable than those at $\gamma=0$ (not shown).

As expected, larger uncertainties in the estimates of rain and $D_{m}$ using the standard DFR take place when the double values occur, as shown in the top-right panels of Figs. 8 and 9. These errors, however, tend to decrease as
$D_{m}$ or the rain rate becomes large; that is, when the DFR$D_{m}$ relationship is single valued. The ambiguities associated with the double solutions not only adversely affect the retrievals of rain rate and $D_{m}$ but also introduce errors into the attenuation corrections. This effect can be seen in the bottom-right panels of Figs. 8 and 9 where errors associated with the $\mathrm{Ku}$ - and Ka-band attenuation corrections become severe enough at the surface to strongly degrade the retrieval estimates, particularly estimates of $D_{m}$. The modified and standard DFR comparisons also have been made at the intermediate gates (not shown). These profiles at intermediate gates behave similarly to those in Figs. 8 and 9 and are bounded by the results estimated at the beginning and end of the profiles. 

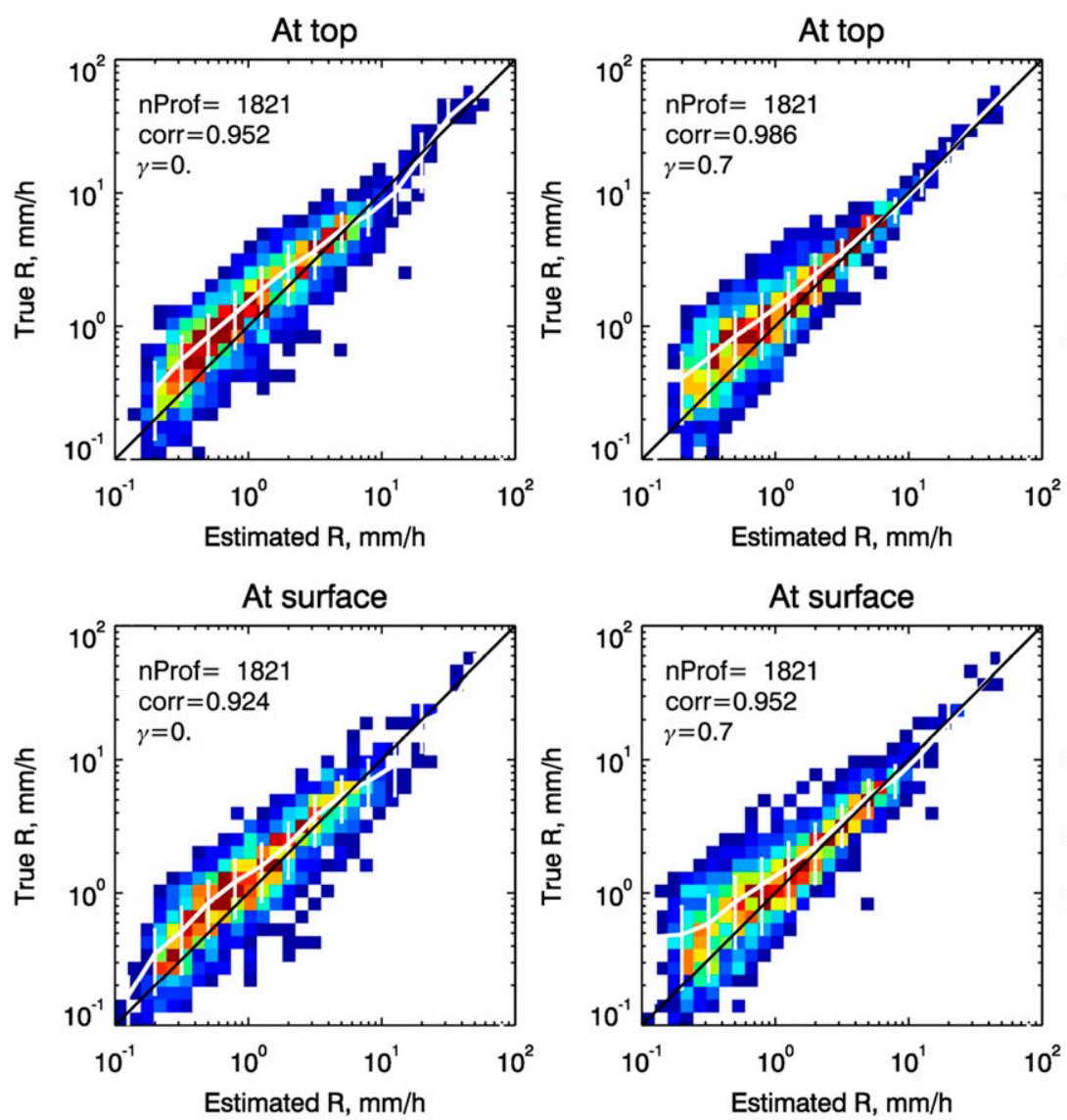

PDF

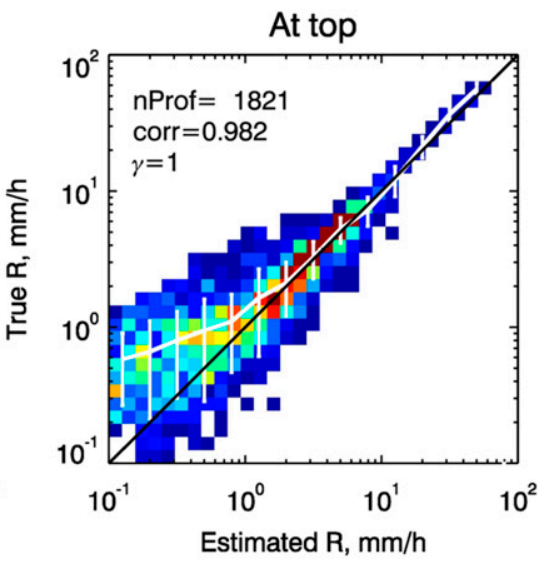

At surface

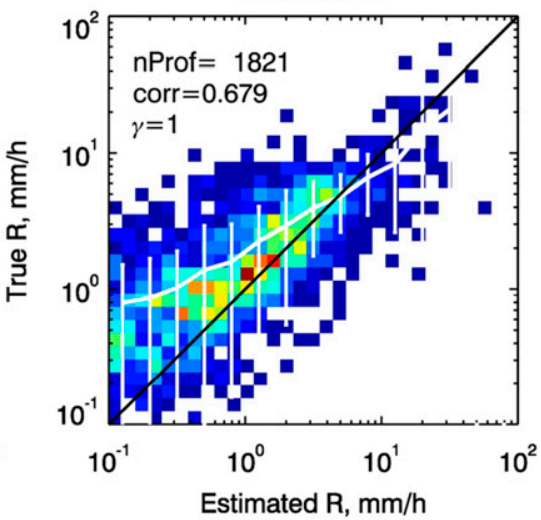

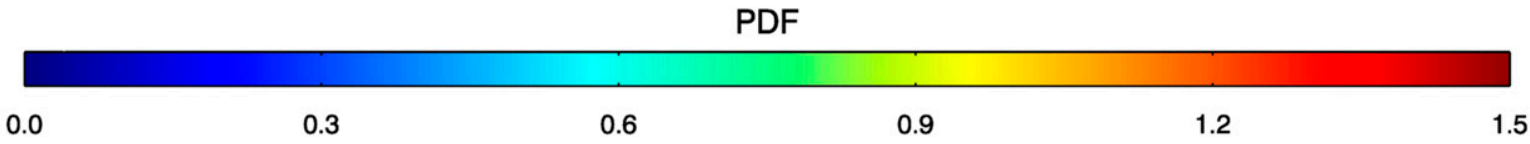

FIG. 9. As in Fig. 8, but for rain rate.

Figures 10 and 11 show the same retrieval results as in Figs. 8 and 9 but for the backward approach. The general behavior of the results obtained from the backward approach largely remains the same as that obtained from the forward approach. Like the forward approach, the modified DFR is superior to the standard DFR method. Because of a reversal of direction of the solution, the last gate of the backward method terminates at the rain top at which the path attenuations from radar are generally small. Unlike the forward method, the accuracy of the PIAs plays a crucial role for the overall performance of the backward procedure.

For the results shown in Figs. 8-11, the DSDs along the vertical column are taken to be nonuniform, that is, partially correlated along the column. If the DSDs are assumed to be fully correlated instead of partially correlated, the rain rate and $D_{m}$ estimates from the modified DFR tend to improve (not shown). This improvement is the result of the fact that fully correlated DSDs are consistent with the assumption that $N_{w}$ is constant over the full profile. Changes in $\sigma_{1}, \sigma_{2}$, and $\sigma_{3}$ values modify the details of the retrievals but not the general characteristics of the results. The functions $p_{1}, p_{2}$, and $p_{3}$ of (13)-(15), serving as constraints on the solutions, play various roles in constraining the results, and their impact on the solutions change with rain intensity. For example, $p_{1}$ constitutes a fairly strong constraint in finding the solutions throughout entire rain-rate range. Function $p_{2}$ is a weak constraint for rain rates less than $1 \mathrm{~mm} \mathrm{~h}^{-1}$, a moderate constraint for rain rates between 1 and $10 \mathrm{~mm} \mathrm{~h}^{-1}$, and a strong constraint for rain rates greater than $10 \mathrm{~mm} \mathrm{~h}^{-1}$. Function $p_{3}$ operates in a manner quite similar to $p_{2}$ in the sense that it is a strong constraint at moderate and heavy rains, and is a slightly stronger constraint than $p_{2}$ for light rain. Overall, the combination of $p_{1}, p_{2}$, and $p_{3}$ provides stronger constraints to the retrieval at moderate-heavy rain rates than for light rain. This is because relative errors of $\delta$ PIA 

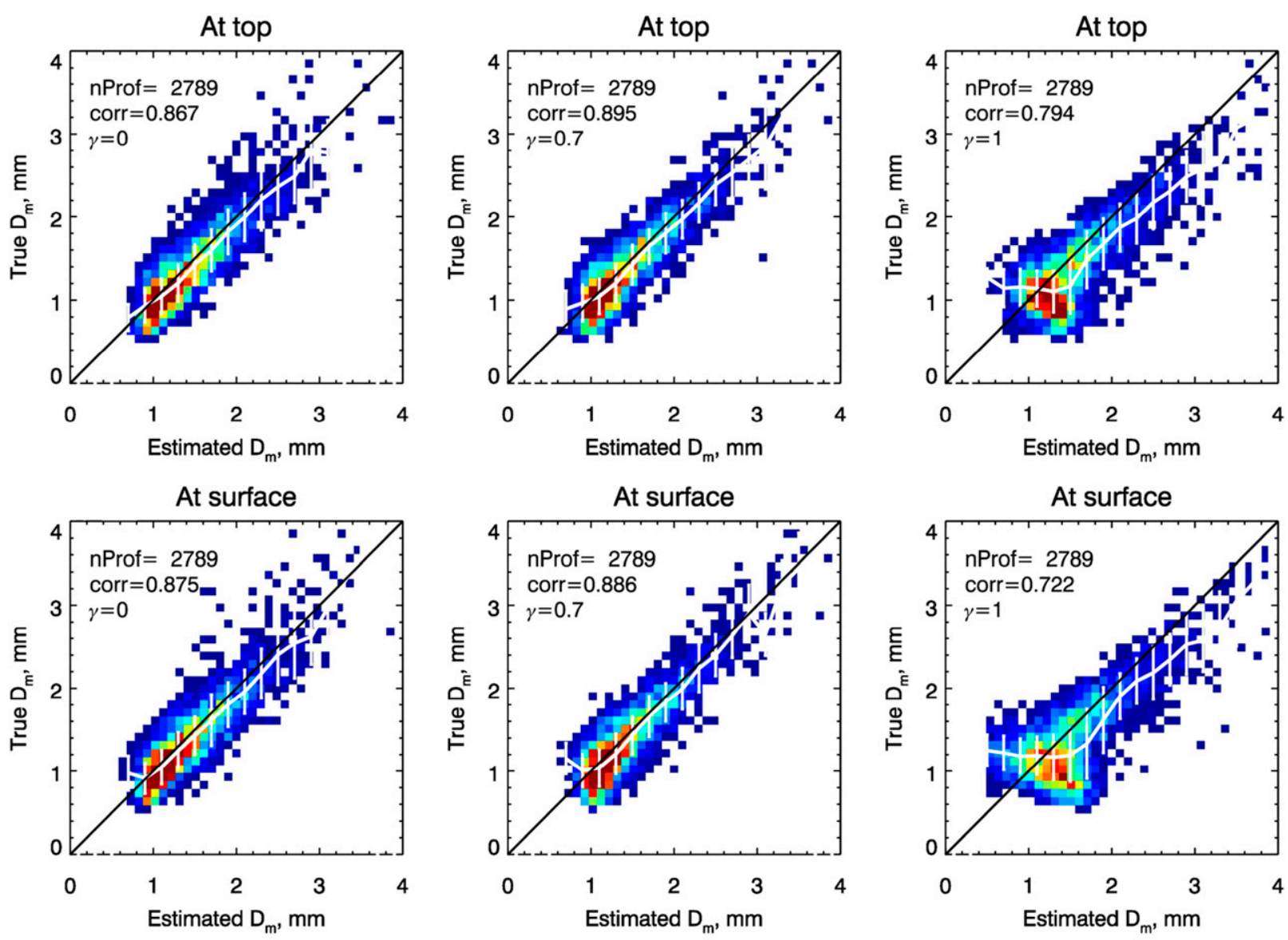

PDF

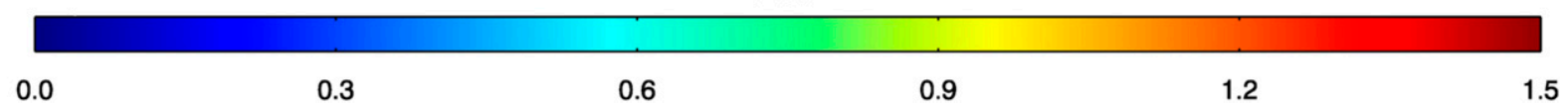

FIG. 10. The 2D probability density functions of $D_{m}$ estimated by the modified DFR with $\gamma$ of (left) 0 and (center) 0.7 as well as (right) the standard DFR $(\gamma=1)$ with the true $D_{m}$ for a range gate at (top) the rain top and (bottom) the surface. The one-to-one lines are given by the black solid curves, while the means and 2 times the standard deviations of the data are given by the white solid curves and vertical bars, respectively. The backward recursive approach is applied in all cases to the nonuniform DSD profiles. Unbiased statistical PIA and $\delta$ PIA models with standard deviations of 2 and $0.8 \mathrm{~dB}$ are assumed, respectively.

[in $p_{2}$ or (13)] become large for the cases where the PIAs are small, which are often associated with the light rain. The Ka-band radar does not provide much additional information if the rain rate is light. In these cases, the backscattering at both $\mathrm{Ku}$ and $\mathrm{Ka}$ band is primarily Rayleigh and the second frequency adds little additional information.

\section{Summary and remarks}

Development of a realistic and physically based framework to evaluate dual-wavelength radar techniques from space is important in assessing the accuracy of current and future retrieval algorithms for the dual-wavelength algorithms. In this study we employ measured DSD data from various NASA-sponsored field campaigns to construct vertical DSD profiles. With these profiles, the measured and attenuation-corrected radar reflectivity factors are simulated along with the corresponding rain rate and DSD parameters. The simulated radar reflectivities and hydrometeor parameters, because they are directly computed from the measured DSD, are considered as truth. Evaluation of the retrieval algorithms is conducted by comparing the hydrometeor parameters derived from the algorithms that use the DSD-simulated measured reflectivities as inputs with those directly computed from the DSD profiles. For the radar simulations the effect of radar random error and calibration bias are not taken into account. 

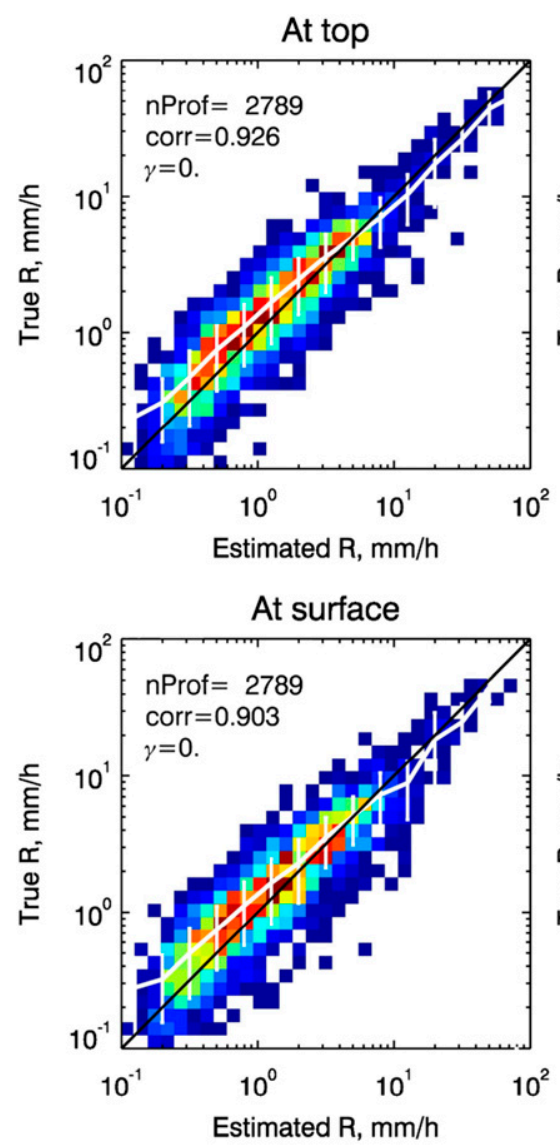

Estimated R, mm/h
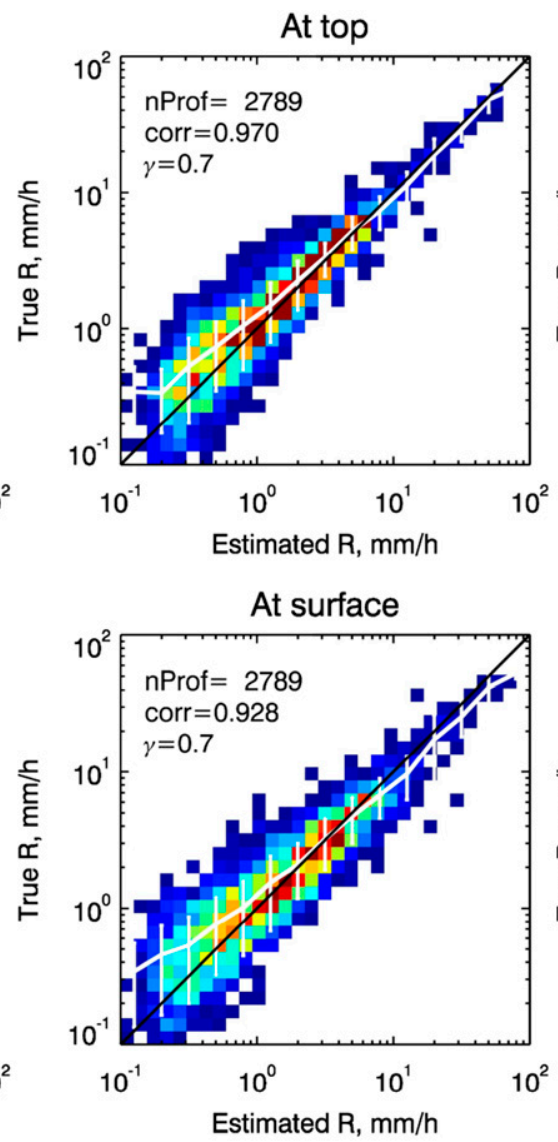

PDF
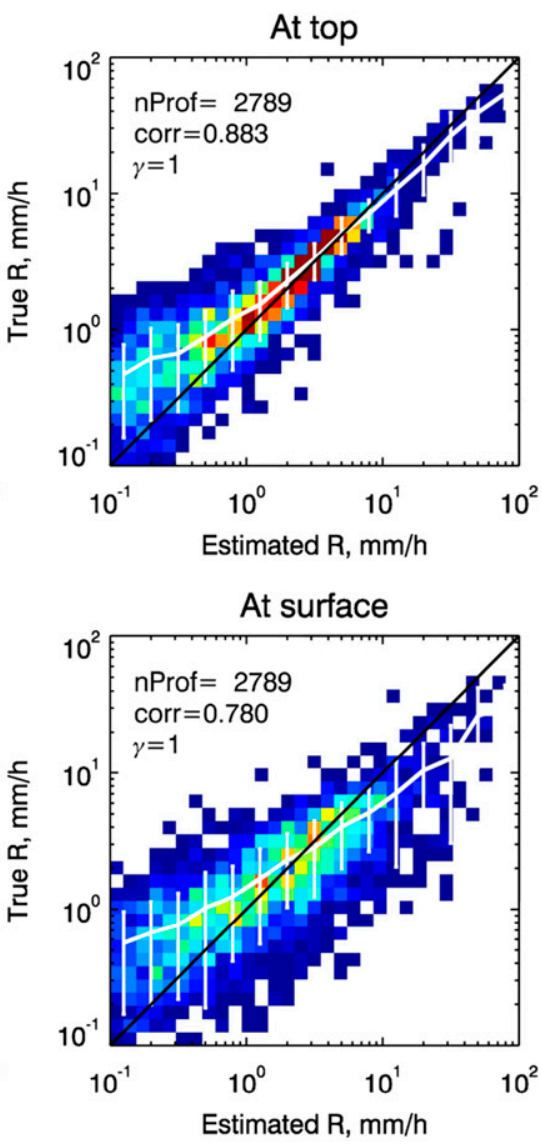

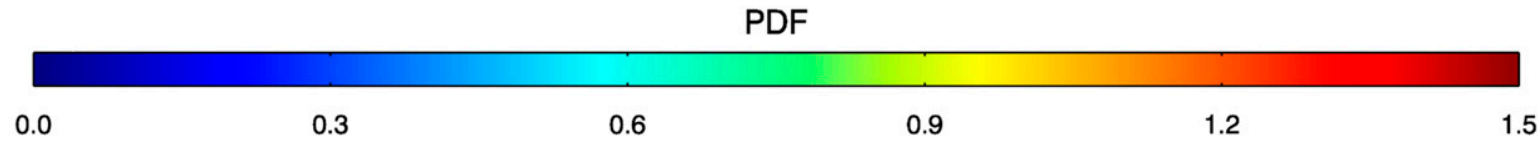

FIG. 11. As in Fig. 10, but for rain rate.

The standard dual-wavelength technique, which relies on DFR $-D_{m}$ relations, has long been considered as a possible means to derive rain and DSD parameters. However, for the $\mathrm{Ku}$ and $\mathrm{Ka}$ combination, negative values of DFR lead to ambiguities in the estimation of $D_{m}$ that can result in large uncertainties in the rain and DSD estimates. If the forward approach is employed, these uncertainties grow larger as the retrieval proceeds deep into the storm as errors are accumulated along the retrieval direction. In an attempt to circumvent the ambiguities in the $D_{m}$ estimation that the standard dual-wavelength method faces, we introduce a modified version of the standard technique, that is, the modified dual-wavelength technique, which partially weights the Ka-band measurements in the radar differentialfrequency-ratio equations. The modified DFR or DFR* can be viewed as the generalized version of the DFR, from which the standard DFR and the single Ku-band equations are the extreme cases with weighting factors of 1 and 0 , respectively.

It has been shown that the modified DFR has a unique relation with $D_{m}$ if the weighting factor (or $\gamma$ ) is less than 0.8 for a given $N_{w}$. Unlike the standard DFR (where $\gamma=1)$, the DFR $*-D_{m}$ relations are dependent on $N_{w}$. The degree to which the DFR* depends on $N_{w}$ depends in turn on the value of $\gamma$. Generally speaking, dependence of the DFR $*-D_{m}$ on $N_{w}$ tends to become weaker as $\gamma$ increases. From the perspective of radar retrieval, the strategy is to choose as large a $\gamma$ as possible as long as a unique solution of $D_{m}$ is achievable. However, a choice of $\gamma$ near 1 leads to an abrupt transition (nonlinear behavior) in the DFR $*-D_{m}$ relations in the range where $D_{m}$ is less than 1 , causing unstable solutions at small values of $D_{m}$. Appropriate choice of $\gamma$ will also depend on some other factors, such as nonuniformity of hydrometeor profiles and model assumptions. 
To apply the modified DFR for the retrieval of rain profiles, following the work of Seto et al. (2016), an optimal profiling retrieval algorithm is first described and then evaluated using the simulated DSD profiles. This is a recursive procedure that infers hydrometeor parameters and makes attenuation correction gate-by-gate along either forward (storm top, toward the surface) or backward (surface, toward the storm top) along the radar beam. As our primary focus is on examining the mechanics of rain retrieval techniques and assessing their retrieval errors, the uncertainties associated with cloud water and melting hydrometeors that often appear atop the rain as well as the issues related to nonuniform beamfilling (NUBF) and multiple scattering are excluded in our evaluation. As such, the attenuation at the first gate for the forward approach (rain top), the gate where the retrieval starts, is assumed to be zero. The PIA is used as the attenuation of the first gate for the backward approach, which is the range gate closest to the surface. The DFR*-based retrievals are carried out $K$ times for a given DSD profile in correspondence with the $K$ equally spaced initialized $\log _{10}\left(N_{w}\right)$. The final $N_{w}$ is then determined as that value that maximizes the product of three probability functions that minimize the differences between the radar measurements and the estimates. Once $N_{w}$ is fixed, the range-profiled rain rate and $D_{m}$ are derived.

It should be pointed out that constant $N_{w}$ along the vertical profile is an approximation, which is true only if the vertical DSD is uniform. For nonuniform DSD profiles, such as the cases studied in the paper, $N_{w}$ varies from gate to gate. The constant $N_{w}$ approximation leads to errors, but these errors are limited by the fact that the natural vertical variations in the DSD are not too strong and the fact that the retrieved $N_{w}$ will be close to a weighted mean of the actual $N_{w}$ values along the path.

Comparisons of the estimates of the rain rate and $D_{m}$ obtained from the modified and standard DFR to the true values indicate that the DFR*-based profiling retrievals are superior to those from the standard DFR. This is true for both forward and backward approaches. As mentioned earlier, attenuations caused by melting layer and cloud water are not included in our evaluation framework, and errors associated with these attenuations are not taken into account. These errors, if considered, will lead to performance degradation for the forward approach but not for the backward method. The backward approach, however, depends on the accuracy of PIA. Highly unreliable PIA will degrade its performance. It is necessary to point out that for the snow retrieval in which the DFR usually has a unique relation to $D_{m}$ from the $\mathrm{Ku}$ - and $\mathrm{Ka}$ band radar, the standard technique is often advantageous over other approaches because it fully accounts for the particle size distribution (PSD) variations in space and time. This is probably true for other frequency pairs that exhibit either a unique DFR- $D_{m}$ relation or muchreduced region of DFR where the double solutions occur.

The benefits of introducing the DFR* are twofold: varying $\gamma$ from 0 to 1 coincides with a transition from the single-frequency case to the full dual-frequency case; the double-valued solutions obtained when $\gamma=1$ can be mitigated by decreasing $\gamma$. Although the DFR* with $\gamma<1$ introduces dependency on $N_{w}$, it facilitates the use of optimal estimation methods similar to that used in the DPR operational retrieval method. Seeking the most reliable and accurate optimization method, though not the main purpose of this study, is important and will therefore be pursued in future studies.

Acknowledgments. This work is supported by Dr. R. Kakar of NASA Headquarters under NASA's Precipitation Measurement Mission (PMM) Grant NNH15ZDA001NPMM. The authors also wish to thank Dr. Ali Tokay from University of Maryland, Baltimore County, for providing DSD data.

\section{REFERENCES}

Adirosi, E., L. Baldini, N. Roberto, P. Gatlin, and A. Tokay, 2016: Improvement of vertical profiles of raindrop size distribution from micro rain radar using $2 \mathrm{D}$ video disdrometer measurements. Atmos. Res., 169, 404-415, https://doi.org/10.1016/j.atmosres.2015.07.002.

Bringi, V. N., and V. Chandrasekar, 2001: Polarimetric Doppler Weather Radar: Principles and Applications. Cambridge University Press, 664 pp.

— G.-J. Huang, V. Chandrasekar, and E. Gorgucci, 2002: A methodology for estimating the parameters of a Gamma raindrop size distribution model from polarimetric radar data: Application to a squall-line event from the TRMM/Brazil campaign. J. Atmos. Oceanic Technol., 19, 633-645, https://doi.org/10.1175/ 1520-0426(2002)019<0633:AMFETP>2.0.CO;2.

Feingold, G., and Z. Levin, 1986: The lognormal fit to raindrop spectra from frontal convective clouds in Israel. J. Climate Appl. Meteor., 25, 1346-1363, https://doi.org/10.1175/15200450(1986)025<1346:TLFTRS > 2.0.CO;2.

Gorgucci, E., G. Scarchilli, V. Chandrasekar, and V. Bringi, 2000: Measurement of mean raindrop shape from polarimetric radar observations. J. Atmos. Sci., 57, 3406-3413, https://doi.org/ 10.1175/1520-0469(2000)057<3406:MOMRSF>2.0.CO;2.

,,,--- and,- 2002 : Estimation of raindrop size distribution parameters from polarimetric radar measurements. J. Atmos. Sci., 59, 2373-2384, https://doi.org/10.1175/15200469(2002)059<2373:EORSDP > 2.0.CO;2.

Grecu, M., L. Tian, W. S. Olson, and S. Tanelli, 2011: A robust dualfrequency radar profiling algorithm. J. Appl. Meteor. Climatol., 50, 1543-1557, https://doi.org/10.1175/2011JAMC2655.1.

Hou, A. Y., and Coauthors, 2014: The Global Precipitation Measurement Mission. Bull. Amer. Meteor. Soc., 95, 701-722, https://doi.org/10.1175/BAMS-D-13-00164.1.

Liao, L., and R. Meneghini, 2005: A study of air/space-borne dualwavelength radar for estimates of rain profiles. Adv. Atmos. Sci., 22, 841-851, https://doi.org/10.1007/BF02918684.

,-- A. Tokay, and L. F. Bliven, 2016: Retrieval of snow properties for Ku- and Ka-band dual-frequency radar. J. Appl. 
Meteor. Climatol., 55, 1845-1858, https://doi.org/10.1175/ JAMC-D-15-0355.1.

Mardiana, R., T. Iguchi, and N. Takahashi, 2004: A dual-frequency rain profiling method without the use of a surface reference technique. IEEE Trans. Geosci. Remote Sens., 42, 2214-2225, https://doi.org/10.1109/TGRS.2004.834647.

Meneghini, R., T. Kozu, H. Kumagai, and W. C. Boncyk, 1992: A study of rain estimation methods from space using dualwavelength radar measurements at near-nadir incidence over ocean. J. Atmos. Oceanic Technol., 9, 364-382, https://doi.org/ 10.1175/1520-0426(1992)009<0364:ASOREM > 2.0.CO;2.

—- H. Kumagai, J. R. Wang, T. Iguchi, and T. Kozu, 1997: Microphysical retrievals over stratiform rain using measurements from an airborne dual-wavelength radar radiometer. IEEE Trans. Geosci. Remote Sens., 35, 487-506, https://doi.org/ 10.1109/36.581956.

, H. Kim, L. Liao, J. A. Jones, and J. M. Kwiatkowski, 2015: An initial assessment of the surface reference technique applied to data from the dual-frequency precipitation radar (DPR) on the GPM satellite. J. Atmos. Oceanic Technol., 32, 2281-2296, https://doi.org/10.1175/JTECH-D-15-0044.1.

Mishchenko, M. I., and L. D. Travis, 1998: Capabilities and limitations of a current FORTRAN implementation of the T-matrix method for randomly oriented, rotation symmetric scatterers. J. Quant. Spectrosc. Radiat. Transfer, 60, 309-324, https://doi.org/10.1016/S0022-4073(98)00008-9.
Rose, C. R., and V. Chandrasekar, 2005: A system approach to GPM dual-frequency retrieval. IEEE Trans. Geosci. Remote Sens., 43, 1816-1826, https://doi.org/10.1109/TGRS.2005.851165.

Seto, S., and T. Iguchi, 2015: Intercomparison of attenuation correction methods for the GPM dual-frequency precipitation radar. J. Atmos. Oceanic Technol., 32, 915-926, https://doi.org/ 10.1175/JTECH-D-14-00065.1.

-, , and T. Oki, 2013: The basic performance of a precipitation retrieval algorithm for the Global Precipitation Measurement mission's single/dual-frequency radar measurements. IEEE Trans. Geosci. Remote Sens., 51, 5239-5251, https://doi.org/10.1109/TGRS.2012.2231686.

— , T. Shimozuma, T. Iguchi, and T. Kozu, 2016: Spatial and temporal variations of mass-weighted mean diameter estimated by GPM/DPR. 2016 IEEE Int. Geoscience and Remote Sensing Symposium (IGARSS), Beijing, China, IEEE, 39383940, https://doi.org/10.1109/IGARSS.2016.7730023.

Thurai, M., G. J. Huang, V. N. Bringi, W. L. Randeu, and M. Schönhuber, 2007: Drop shapes, model comparisons, and calculations of polarimetric radar parameters in rain. J. Atmos. Oceanic Technol., 24, 1019-1032, https://doi.org/ 10.1175/JTECH2051.1.

Ulbrich, C. W., 1983: Natural variations in the analytical form of the raindrop size distribution. J. Climate Appl. Meteor., 22, 1764-1775, https://doi.org/10.1175/1520-0450(1983)022<1764: NVITAF $>2.0 . \mathrm{CO} ; 2$. 